\title{
EXTREMAL LENGTH OF WEAK HOMOLOGY CLASSES ON RIEMANN SURFACES ${ }^{1}$
}

\author{
BURTON RODIN
}

1. The square integrable harmonic differentials on a Riemann surface $W$ form a Hilbert space $\Gamma_{h}$. Let $\Gamma_{x}$ be a closed subspace of $\Gamma_{h}$. Let $c$ be a 1-chain on $W$. There exists a unique element $\psi(c) \in \Gamma_{x}$ with the property $\int_{c} \omega=(\omega, \psi(c))$ for all $\omega \in \Gamma_{x}$. We refer to $\psi(c)$ as the $\Gamma_{x^{-}}$ reproducing differential for $c$. Accola [1] has shown that if $c$ is a cycle, then the extremal length of the homology class of $c$ is equal to the square of the norm of the $\Gamma_{h}$-reproducing differential for $c$ (cf. also [3]). Two specific problems raised by Accola's result are the following. For the important subspaces $\Gamma_{x}$, does the norm of the $\Gamma_{x^{-}}$ reproducing differential for a cycle have an extremal length interpretation? Secondly, we may ask for a family of curves associated with a 1-chain $c$, not necessarily a cycle, whose extremal length gives the norm of the $\Gamma_{h}$-reproducer for $c$. (By Abel's theorem, the vanishing of the norm of this reproducer implies that $\partial c$ is a principal divisor.)

In the present paper we give an answer to the first question for the subspace $\Gamma_{h s e}$. Theorem 1 states that an associated geometric configuration is the weak homology class of $c .^{2}$

2. Let $\Gamma_{x}$ be a closed subspace of $\Gamma_{h}$ such that $\Gamma_{x}=\bar{\Gamma}_{x}$. We say that two cycles $c_{1}$ and $c_{2}$ are $\Gamma_{x}$-homologous, denoted by $c_{1} \approx c_{2}\left(\bmod \Gamma_{x}\right)$, if $\int_{c_{1}-c_{2}} \omega=0$ for all $\omega \in \Gamma_{x}$. Denote the $\Gamma_{x}$-homology class of a cycle $c$ by $c^{x}$.

An invariant expression $\rho(z)|d z|$ with $\rho$ a nonnegative and lower semicontinuous function is called a linear density. The $\rho$-area is

$$
A(\rho)=\iint_{W} \rho^{2} d x d y .
$$

The $\rho$-length of a family $\mathcal{F}$ of arcs is

Received by the editors February 27, 1963.

1 This research was supported by the Air Force Office of Scientific Research.

2 Another special case of the first question has been settled by A. Marden. He has shown that a geometric configuration for the subspace $\Gamma_{h 0}$ (notation as in [2]) is the set of relative, i.e., possibly infinite cycles which are weakly homologous to $c$ [ $A n$ extremal length problem and the bilinear relation on open Riemann surfaces, doctoral dissertation, Harvard University, May, 1962]. 


$$
L(\mathcal{F}, \rho)=\inf \left\{\int c \rho|d z|: c \in \mathcal{F}\right\} .
$$

The extremal length of $\mathcal{F}$ is

$$
\lambda(\mathcal{F})=\sup _{\rho} L(F, \rho)^{2} / A(\rho) .
$$

Lemma 1. Let $c$ be a cycle on $W$. Let $\lambda\left(c^{x}\right)$ be the extremal length of all cycles $\Gamma_{x}$-homologous to $c$. Let $\psi$ be the $\Gamma_{x}$-reproducing differential for $c$. Then $\lambda\left(c^{x}\right) \geqq\|\psi\|^{2}$.

Proof. Let $\rho_{0}|d z|$ be the linear density $\left|\psi+i \psi^{*}\right|$. Then $A\left(\rho_{0}\right)$ $=\|\psi\|^{2}$. For $\delta \in c^{x}$ we have $\int_{\delta} \rho_{0}|d z| \geqq\left|\int_{\delta} \psi\right|=\left|\int_{c} \psi\right|=\|\psi\|^{2}$, and the desired inequality follows. We have used the fact that $\Gamma_{x}=\bar{\Gamma}_{x}$ which implies that $\psi$ is real.

3. We shall prove a converse of Lemma 1 for $\Gamma_{x}=\Gamma_{h s e}$. First note that $c_{1} \approx c_{2}\left(\bmod \Gamma_{h s e}\right)$ if and only if $c_{1}-c_{2}$ is weakly homologous to zero. In fact, $c_{1} \approx c_{2}\left(\bmod \Gamma_{h s e}\right)$ holds exactly when $c_{1}-c_{2}$ is a dividing cycle (see Theorem V.20D of [2]), which in turn is equivalent to being weakly homologous to zero (see Theorem I.32C, ibid.).

Let $\Omega$ be the interior of a compact bordered Riemann surface $\bar{\Omega}$. Let $c$ be a cycle in $\Omega$ and $\psi_{\Omega}$ the $\Gamma_{h s e}$-reproducer for $c$. Let $L_{1}$ be the normal operator for the canonical partition of $\partial \Omega$ (cf. [2]). Corollary 6 of [4] shows that $\psi_{\Omega}=(2 \pi)^{-1} d p^{*}$ where $p$ is a harmonic function on $W-c$ and satisfies $p=L_{1} p$ in a boundary neighborhood of $\Omega$. Thus $p$ is constant on each contour $\beta_{i}$ of $\bar{\Omega}$ and $\int_{\beta_{i}} d p^{*}=0$. If $\delta$ is a cycle on $\Omega$ then $(2 \pi)^{-1} \int_{\delta} d p$ is an integer equal to the intersection number $\delta \times c$. Furthermore, the $\Gamma_{h s e}$-reproducer for an open surface $W$ is the limit of $\psi_{\Omega}$ for exhausting canonical subregions $\Omega \rightarrow W$.

In the course of the following proofs we find occasion to use arguments similar to those expressed or implied in Accola [1]. For convenience to the reader we repeat his reasoning in such situations.

Lemma 2. Let $c$ be a cycle on a compact bordered Riemann surface and

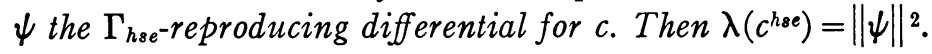

Proof. Denote the bordered surface by $\bar{\Omega}$ and its interior by $\Omega$. Let the contours be $\beta_{1}, \cdots, \beta_{n}$. Let $V$ be the equivalence class of $\beta_{1}$ in the sense of Accola [1]. That is, $V$ is the set of points in $\bar{\Omega}$ which can be joined to a point of $\beta_{1}$ by an arc $\delta$ for which $\int_{\delta} \psi^{*}$ is an integer. $v$ is a closed set which is locally a level curve of a harmonic function. Let $\Omega-V=R_{1} \cup \ldots \cup R_{m}$ be a decomposition into components. Each $R_{\nu}$ is a finite surface. The points of $V$ serve as a piecewise analytic 
border for $R_{\nu}$ if we allow multiplicities for the prime ends. $R_{\nu}$ together with its boundary shall be denoted by $R_{\nu}^{*}$. We may omit the details of this construction but remark that there is an analytic mapping $R_{\nu}^{*} \rightarrow \bar{\Omega}$ which restricts to the identity on $R_{\nu}$. By means of this mapping we refer to points of $\partial R_{\nu}^{*}$ as belonging to $\mathcal{V}$ or $\partial \bar{\Omega}$.

$\psi^{*}$ is exact on $R_{\nu}$, say $\psi^{*}=d p_{\nu}$, and we know that $p_{\nu}$ extends harmonically to $R_{\nu}^{*}$. Since each point of $\partial R_{\nu}^{*}$ belongs to $U$ or $\partial \bar{\Omega}$ we see that $p_{\nu}$ is constant on each component of $\partial R_{\nu}^{*}$. We adjust $p_{\nu}$ so that the smallest such constant on $V$ is zero. Now let $\rho_{\nu}$ be the collection of those boundary components of $R_{\nu}^{*}$ on which $p_{\nu}=0, \sigma_{\nu}$ those on which $p_{\nu}=1$, and let $\tau_{\nu}$ contain the remaining ones. We orient them so that $\partial R_{\nu}^{*}=\rho_{\nu}+\sigma_{\nu}+\tau_{\nu}$. The points of $\rho_{\nu}$ and $\sigma_{\nu}$ belong to $\nu$, those of $\tau_{\nu}$ belong to $\partial \bar{\Omega}-\mathcal{V}$. Let us show that if $\tau_{\nu}$ contains a point $t$ of some $\beta_{k}$ then it must contain all of $\beta_{k}$. For $t_{1} \in \beta_{1}, \int_{t_{1}}^{t} \psi^{*}$ is not an integer and since $\psi^{*}=0$ along $\beta_{k}$, it follows that $\beta_{k}$ has a connected neighborhood disjoint from $v$. This neighborhood must be in $R_{\nu}$, hence $\beta_{k} \subset \tau_{\nu}$.

By means of the mapping $R_{\nu}^{*} \rightarrow \bar{\Omega}$, we consider $\sigma_{\nu}$ as a 1-chain on $\bar{\Omega}$ and claim that $c \approx \Sigma_{\nu} \sigma_{\nu}\left(\bmod \Gamma_{h s e}\right)$. Let $\omega \in \Gamma_{h s e}$ and assume that $\omega$ extends harmonically to $\bar{\Omega}$. Then $\int_{c} \omega=(\omega, \psi)=\Sigma_{\nu}\left(\omega, d p_{\nu}^{*}\right)$. By partial integration we have $\left(\omega, d p_{\nu}^{*}\right)=\iint_{R_{\nu}} d p_{\nu} \wedge \omega=\int_{\sigma_{\nu}} \omega+\int_{\tau_{\nu}} p_{\nu} \omega$. We have seen that $\tau_{\nu}$ is a union of contours $\beta_{\nu_{1}}, \cdots, \beta_{\nu_{k}}$ on each of which $p_{\nu}$ is a constant. Since $\omega$ is semiexact we obtain $\int_{c} \omega=\int_{\Sigma_{\nu} \sigma_{\nu}} \omega$. It follows that $c-\Sigma_{\nu} \sigma_{\nu}$ is a dividing cycle.

The function $p_{\nu}$ in $R_{\nu}$ has boundary values 0 on $\rho_{\nu}, 1$ on $\sigma_{\nu}$ and constants $k_{\nu_{\mu}}$ on $\beta_{\nu_{\mu}}$, those contours of $\bar{\Omega}$ which make up $\tau_{\nu}$. These constants must satisfy $0<k_{\nu_{\mu}}<1$ in order for the flux condition $\int_{\beta_{\nu \mu}} d p_{\nu}^{*}=0$ to hold. Consequently, for $s \in(0,1)$ the level curves $\sigma_{\nu}(s)=p_{\nu}^{-1}(s)$ are compact and weakly homologous to $\sigma_{\nu}$, except for the finite number of values $s=k_{\nu \mu}$. Let $\sigma(s)=\Sigma_{\nu} \sigma_{\nu}(s)$. Let $\rho$ be a linear density on $\Omega$. Then for almost all $s \in(0,1)$

$$
L^{2}\left(\rho, c^{h s e}\right) \leqq\left(\int_{\sigma(s)} \rho \psi\right)^{2} \leqq \int_{\sigma(s)} \rho^{2} \psi \int_{\sigma(s)} \psi=\|\psi\|^{2} \int_{\sigma(s)} \rho^{2} \psi .
$$

Integrating over $s \in(0,1)$ we obtain

$$
L^{2}\left(\rho, c^{h s e}\right) \leqq\|\psi\|^{2} A(\rho) .
$$

This, together with the opposite inequality of Lemma 1, completes the proof.

4. Theorem. Let $W$ be an open Riemann surface. Let $\psi$ be the $\Gamma_{h s e}$-reproducing differential for a cycle $c$ on $W$. Then $\|\psi\|^{2}$ gives the extremal length of all cycles weakly homologous to $c$. 
Proof. Let $\Omega$ be a canonical subregion of $W$ and $\psi_{\Omega}$ the $\Gamma_{\text {hee }}(\Omega)$ reproducing differential for $c$. Thanks to the above lemmas we have

$$
\lambda\left(c^{\text {hse }}\right) \geqq\|\psi\|^{2}=\lim _{\Omega \rightarrow W}\left\|\psi_{\Omega}\right\|_{\Omega}^{2}=\lim _{\Omega \rightarrow W} \lambda_{\Omega}\left(c^{\text {hse }}\right) \geqq \lambda\left(c^{\text {hes }}\right) .
$$

\section{BIBLIOGRAPHY}

1. R. D. M. Accola, Differentials and extremal length on Riemann surfaces, Proc. Nat. Acad. Sci. U.S.A. 46 (1960), 540-543.

2. L. Ahlfors and L. Sario, Riemann surfaces, Princeton Univ. Press, Princeton, N. J., 1960.

3. C. Blatter, Une inégalité de géométrie différentielle, C. R. Acad. Sci. Paris 250 (1960), 1167.

4. B. Rodin, Reproducing kernels and principal functions, Proc. Amer. Math. Soc. 13 (1962), 982-992.

HARVARD UNIVERSITY 